\title{
Safety of a Novel Dialyzer Containing a Fluorinated Polyurethane Surface-Modifying Macromolecule in Patients with End-Stage Kidney Disease
}

\author{
Jill M. Meyer ${ }^{a} \quad$ Dylan Steer ${ }^{b} \quad$ Lisa A. Weber ${ }^{c} \quad$ Abeer A. Zeitone ${ }^{d}$ \\ Mayuri Thakuriad Chiang-Hong Ho ${ }^{d}$ Shakil Aslam ${ }^{d}$ Claudy Mullon ${ }^{d}$ \\ Robert J. Kossmann ${ }^{\text {d }}$ \\ aCalifornia Institute of Renal Research, Chula Vista, CA, USA; 'balifornia Institute of Renal Research, San Diego, CA, \\ USA; 'Research Management Inc./Kansas Nephrology Research, Wichita, KS, USA; ${ }^{\circ}$ Fresenius Medical Care North \\ America, Renal Therapies Group, Waltham, MA, USA
}

\section{Keywords \\ Dialyzer - Chronic hemodialysis · $\beta 2$-Microglobulin . \\ Surface-modifying macromolecule}

\begin{abstract}
Background: By inhibiting the adsorption of protein and platelets, surface-modifying macromolecules (SMMs) may improve the hemocompatibility of hemodialyzers. This trial aims to assess the performance and safety of a novel dialyzer with a fluorinated polyurethane SMM, Endexo ${ }^{\mathrm{TM}}$. Methods: This prospective, sequential, multicenter, open-label study (NCT03536663) was designed to meet regulatory requirements for clinical testing of new hemodialyzers, including assessment of the in vivo ultrafiltration coefficient (Kuf). Adults prescribed thrice-weekly hemodialysis were eligible for enrollment. After completing 12 hemodialysis sessions with an Optiflux ${ }^{\oplus}$ F160NR dialyzer, patients received 38 sessions with the dialyzer with Endexo. Evaluated parameters included the in vivo Kuf of the dialyzer with Endexo extent of removal of urea, albumin, and $\beta 2$-microglobulin ( $\beta 2 M)$, as well as complement activation. Results: Twenty-three patients received 268 hemodialysis treatments during the Op-
\end{abstract}

tiflux period, and 18 patients received 664 hemodialysis treatments during the Endexo period. Three serious adverse events were reported, and none of them were considered device related. No overt complement activation was observed with either dialyzer. Both dialyzers were associated with comparable mean increases in serum albumin levels from pre-to posthemodialysis (Optiflux:7.9\%; Endexo: 8.0\%). These increases can be viewed in the context of a mean increase in hemoglobin of approximately $5 \%$ and a mean ultrafiltration volume removed of approximately $2.2 \mathrm{~L}$. The corrected mean $\beta 2 \mathrm{M}$ removal rate was $47 \%$ higher during the Endexo period (67.73\%). Mean treatment times (208 vs. $205 \mathrm{~min}$ ), blood flow rates ( $447.7 \mathrm{vs} .447 .5 \mathrm{~mL} / \mathrm{min}$ ), dialysate flow rates $(698.5$ vs. $698.0 \mathrm{~mL} / \mathrm{min})$, urea reduction ratio $(82$ vs. $81 \%)$, and spKt/V (2.1 vs. 1.9$)$ were comparable for the Endexo and Optiflux periods, respectively. The mean (SD) Kuf was 15.85 (10.33) $\mathrm{mL} / \mathrm{h} / \mathrm{mm} \mathrm{Hg}$ during the first use of the dialyzer with Endexo (primary endpoint) and 16.36 (9.92) $\mathrm{mL} / \mathrm{h} / \mathrm{mm} \mathrm{Hg}$ across the Endexo period. Conclusions: The safety of the novel dialyzer with Endexo was generally comparable to the Optiflux dialyzer, while exhibiting a higher $\beta 2 \mathrm{M}$ removal rate.

(c) 2021 The Author(s).

Published by S. Karger AG, Basel karger@karger.com www.karger.com/bpu

Karger $\stackrel{\text { ' }}{=}$

BOPEN ACCESS
(C) 2021 The Author(s)

Published by S. Karger AG, Basel

This is an Open Access article licensed under the Creative Common Attribution-NonCommercial-4.0 International License (CC BY-NC) (http://www.karger.com/Services/OpenAccessLicense), applicable to the online version of the article only. Usage and distribution for commercial purposes requires written permission. 


\section{Introduction}

Globally, it is estimated that $>3.7$ million individuals will receive renal replacement therapy for ESKD in 2020 [1], and that most of those patients will receive maintenance hemodialysis. In the absence of an ideal dialyzer one capable of clearing all uremic toxins while allowing retention of beneficial plasma components and molecules - the selection of a dialyzer should be tailored to the needs of the patient and the dialysis setting [2].

Endexo $^{\mathrm{TM}}$, a novel fluorinated polyurethane surfacemodifying macromolecule (SMM), is designed to inhibit the adsorption of protein and platelets [3]. The Endexo additive is currently approved for use in peripherally inserted central catheters and dialysis catheters [3-5]. It is hypothesized that incorporating Endexo into dialyzer fibers will improve hemocompatibility and aid in the development of heparin-sparing hemodialysis. Dialyzers with Endexo are high-flux, single-use dialyzers with a surface area of $1.5 \mathrm{~m}^{2}$ manufactured by mixing polysulfone, polyvinylpyrrolidone, and the Endexo SMM. Relative to a control polysulfone membrane, the membrane exhibits a slight increase in hydrophobicity in the inner lumen and up to a $40 \%$ increase in the outer lumen [6]. The zeta potential in the inner lumen is also reduced (vs. a polysulfone membrane). In vitro simulations with the dialyzer with Endexo suggest a smaller impact on platelet count reduction and less platelet activation than is observed with the control dialyzer. This clinical study was conducted to meet US regulatory guidance for clinical performance testing of a new dialyzer [7] and represents the first in-human use of a dialyzer with Endexo.

\section{Materials and Methods}

\section{Study Design and Participants}

This prospective, sequential, open-label study (NCT03536663) was designed to align with the US Food and Drug Administration (FDA) guidance on performance testing of new dialyzers [7]. The study was conducted at 3 US hemodialysis centers from August 2018 through April 2019. Eligible patients were $\geq 22$ years of age, prescribed in-center thrice-weekly hemodialysis for $\geq 180$ days, and had received hemodialysis treatments with the Optiflux ${ }^{\circledR}$ F160NR dialyzer (manufactured by Fresenius Medical Care North America, Waltham, MA) continuously for $\geq 30$ days prior to entering the study. Other inclusion criteria included a prescribed hemodialysis treatment time of 3-4.5 h, stable systemic anticoagulation with heparin for $\geq 2$ weeks, hemoglobin concentration of $\geq 9 \mathrm{~g} / \mathrm{dL}$, and a platelet count of $\geq 100,000 / \mathrm{mm}^{3}$. Patients were excluded from participation if they received hemodialysis with a citric acid concentrate.
Following a screening period of $\leq 4$ weeks, patients underwent 12 hemodialysis treatments with the Optiflux F160NR dialyzer over 4 weeks (Optiflux period). The Endexo period consisted of 38 hemodialysis treatments on the dialyzer with Endexo approximately 3 times a week over 13 weeks. After the last scheduled hemodialysis treatment in the Endexo period, patients resumed hemodialysis with their previously prescribed dialyzer and attended an in-center follow-up visit within 1 week (Fig. 1). This study was conducted in accordance with the Principles of the International Council for Harmonization (ICH) Guideline for Good Clinical Practice (GCP; E6), with approval by an independent Institutional Review Board (Advarra Inc., Columbia, MD, USA) and adheres to applicable CONSORT guidelines.

\section{Endpoints}

The selection of endpoints for the present study was based on regulatory guidance [7]. The primary endpoint was the in vivo ultrafiltration coefficient (Kuf) of the dialyzer with Endexo during the first study use (visit 13) at $15( \pm 5)$ min after the recorded start of hemodialysis. This endpoint was derived from machine readings of transmembrane pressure (TMP) and ultrafiltration rate (UFR) and calculated as UFR/TMP.

Secondary endpoints included the extent of urea removal (i.e., urea reduction ratio [URR]) and the single-pool Kt/V calculated from pre- and posthemodialysis blood urea nitrogen concentrations (spKt/V) for the first study use of each dialyzer (Fig. 1). Albumin and $\beta 2$-microglobulin $(\beta 2 \mathrm{M})$ concentrations were evaluated before and after hemodialysis treatments for the first study use of each dialyzer. Adverse events (AEs) were coded using Medical Dictionary for Regulatory Activities (MedDRA), Version 20.0, and evaluated as secondary endpoints.

Consistent with regulatory guidance [7], complement activation (i.e., C3a, C5a, and sC5b-9) was assessed before hemodialysis and $30( \pm 10)$ min after the recorded start of hemodialysis during the first study use of each dialyzer. At the end of each hemodialysis session, thrombus scoring was performed for each dialyzer using a previously published graded scoring method $[8,9]$. Finally, data on hemodialysis treatment-related medications (e.g., erythropoiesis-stimulating agents [ESAs], iron, and calcitriol), clinical laboratory values, physical examination, and vital signs were recorded.

\section{Analysis}

A planned sample size of 15-24 participants was aligned with regulatory guidance recommending a minimum of 12 patients who each receive $\geq 36$ treatments with the investigational dialyzer [7]. The safety population included all individuals who had $\geq 1$ treatment on the dialyzer with Endexo. All other endpoints were evaluated in the analysis population, defined as all individuals with $\geq 36$ hemodialysis treatments on the dialyzer with Endexo. Continuous endpoints are described with descriptive statistics, whereas categorical endpoints are described with frequency and percentage. Missing values were not imputed.

To characterize changes in serum albumin and $\beta 2 \mathrm{M}$ associated with each dialyzer, the relative percentage change was calculated. Percentage changes were calculated using corrected posthemodialysis $\beta 2 \mathrm{M}$ concentrations based on the work by Bergström and Wehle [10]. In addition to descriptive statistics of complement measures, the relative percentage change (from before dialysis to $30 \mathrm{~min}$ after initiation) was calculated. Thrombus formation is presented as the number of patients and treatments in each period 


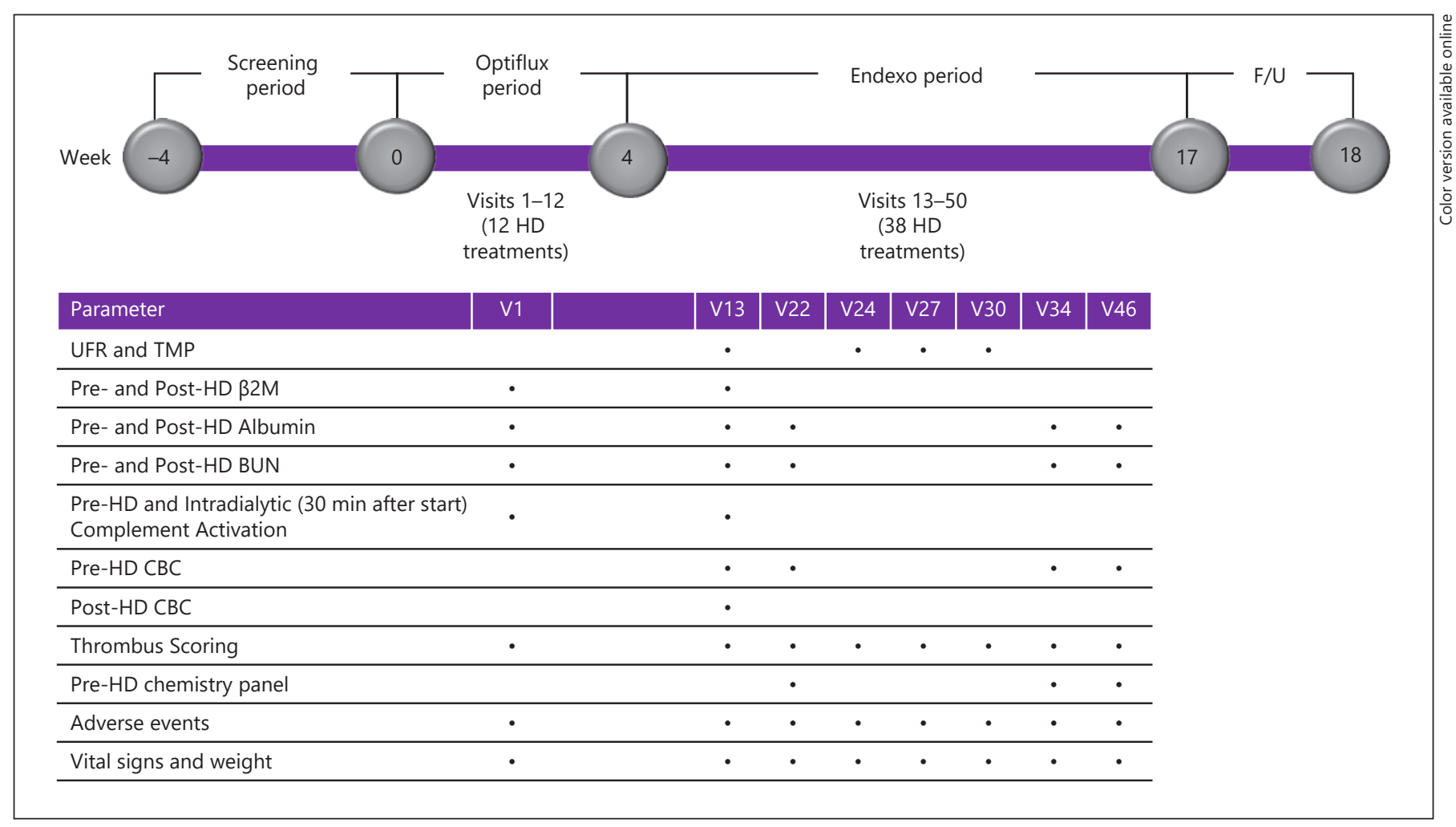

Fig. 1. Study design and select schedule of assessments. Not all visits and assessments are listed. F/U, follow-up; HD hemodialysis; BUN, blood urea nitrogen; $\beta 2 \mathrm{M}$, beta- 2 microglobulin; $\mathrm{CBC}$, complete blood count; TMP, transmembrane pressure; UFR, ultrafiltration rate; V, visit; AEs, adverse events.

for each grade category (i.e., grade 1 [clear] through 4 [fully clotted]) and as the proportion of treatments with full dialyzer clotting for each patient in each period. AEs and device-related events per treatment were calculated.

\section{Results}

Twenty-six adults provided informed consent and were evaluated for study entry; 23 patients were enrolled and received $\geq 1$ hemodialysis treatment with the Optiflux dialyzer. Per protocol, 4 patients were withdrawn from the study prior to the completion of the Optiflux period because they missed a hemodialysis treatment. These 4 patients were aged 62-79 years and included 2 men and 2 women. ESKD was secondary to diabetes $(n=3)$ and hypertension/large-vessel disease $(n=1)$. Of the 19 patients who completed the Optiflux period, one (a 28-yearold female with ESKD secondary to glomerulonephritis) missed her first treatment on the dialyzer with Endexo (visit 13) and was withdrawn from the study. The safety population consisted of the 18 patients who received $\geq 1$ hemodialysis session during the Endexo period. As a result of missing multiple hemodialysis treatments during the Endexo period secondary to a localized foot infection (judged to be unrelated to the device), 1 participant was withdrawn from the study per protocol. As such, $17 \mathrm{pa}-$ tients made up the analysis population.

\section{Baseline Characteristics and Hemodialysis Treatment \\ Delivered}

The median age of patients in the safety population $(N=18)$ was 63.5 years (range $27-87)$. The population was mostly female (78\%) and identified as white (67\%). Diabetes was the most common cause of ESKD, accounting for $61 \%$ of cases; hypertension/large-vessel disease, cystic/hereditary/congenital diseases, and glomerulonephritis accounted for 22,11 , and $6 \%$ of cases, respectively.

Initial hemodialysis prescriptions had blood flow rates that ranged from 300 to $500 \mathrm{~mL} / \mathrm{min}$, dialysate flow rates of $450-800 \mathrm{~mL} / \mathrm{min}$, and treatment durations of $180-243$ min. In the Optiflux period, 23 patients received a total of 
Table 1. Delivered hemodialysis for the analysis population $(N=17$ completers $)$

\begin{tabular}{lcr}
\hline Measure & Optiflux & \multicolumn{1}{l}{ Endexo } \\
\hline Blood pump flow rate, mL/min & $447.5(32.3)$ & $447.7(37.8)$ \\
Dialysate flow rate, mL/min & $698.0(60.7)$ & $698.5(62.9)$ \\
Treatment time, min & $205.2(19.1)$ & $207.5(20.5)$ \\
Blood volume processed, $\mathrm{L}$ & $82.6(8.1)$ & $82.8(10.7)$ \\
Ultrafiltration volume removed, $\mathrm{mL}$ & $2,156.9(632.6)$ & $2,187.1(739.0)$ \\
\hline
\end{tabular}

Data presented as mean $(\mathrm{SD})$.

Table 2. Ultrafiltration coefficient data during hemodialysis sessions in the Endexo period for the analysis population

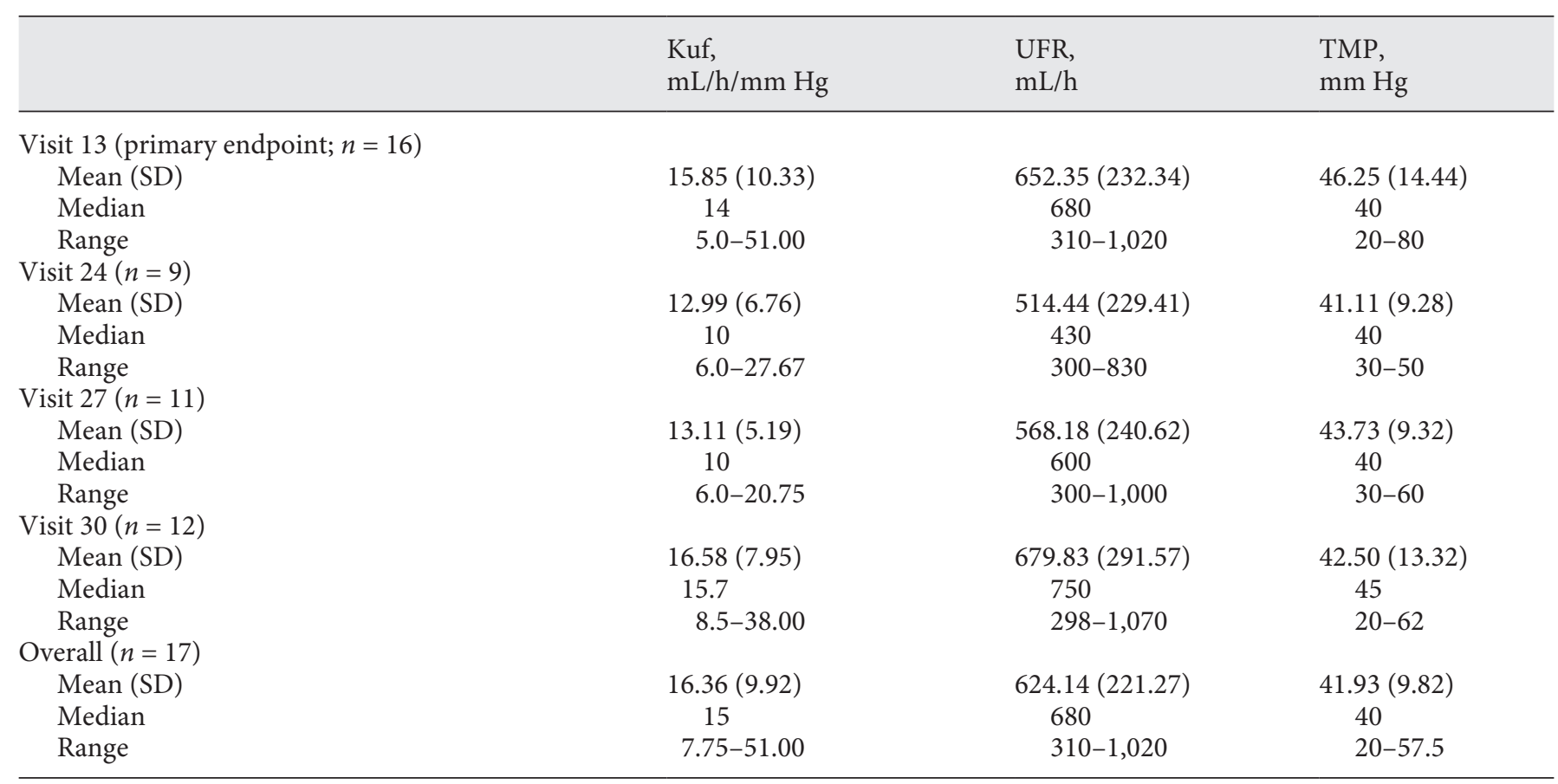

Kuf, ultrafiltration coefficient calculated as UFR/TMP; TMP, transmembrane pressure; UFR, ultrafiltration rate.

268 hemodialysis treatments, and 18 participants received 664 hemodialysis treatments in the Endexo period. In the analysis population, delivered hemodialysis treatment parameters were comparable across treatment periods (Table 1).

\section{Primary and Secondary Endpoints}

The mean (SD) in vivo Kuf of the dialyzer with Endexo at visit 13 (primary endpoint) was $15.85(10.33) \mathrm{mL} / \mathrm{h} /$ $\mathrm{mm} \mathrm{Hg}$ (Table 2). At the same session, mean (SD) dialysis adequacy (i.e., spKt/V) was 1.98 (0.56), and URR was 79.59\% (12.15). Using data across the entirety of each treatment period, the mean (SD) spKt/V for the Optiflux dialyzer and dialyzer with Endexo was 1.94 (0.3) and 2.09
(0.41), respectively. Treatment with the dialyzers was associated with comparable mean (SD) URRs in the Optiflux (80.81\% [4.33]) and Endexo (81.87\% [5.91]) periods.

Mean (SD) predialysis concentrations of $\beta 2 \mathrm{M}$ were similar at visit 1 (Optiflux) and visit 13 (Endexo): 32.29 (8.37) and $32.9(6.55) \mu \mathrm{g} / \mathrm{mL}$, respectively. As shown in Figure 2, hemodialysis treatment with the dialyzer with Endexo resulted in corrected mean (SD) $\beta 2 \mathrm{M}$ removal rates that were $47 \%$ higher than those observed with the Optiflux dialyzer. Mean (SD) predialysis serum albumin levels were comparable for all visits: $3.97(0.34) \mathrm{g} / \mathrm{dL}$ (visit 1; Optiflux), 3.95 (0.24) g/dL (visit 13; Endexo), 3.91 (0.21) g/dL (visit 22; Endexo), 3.94 (0.25) g/dL (visit 34; 


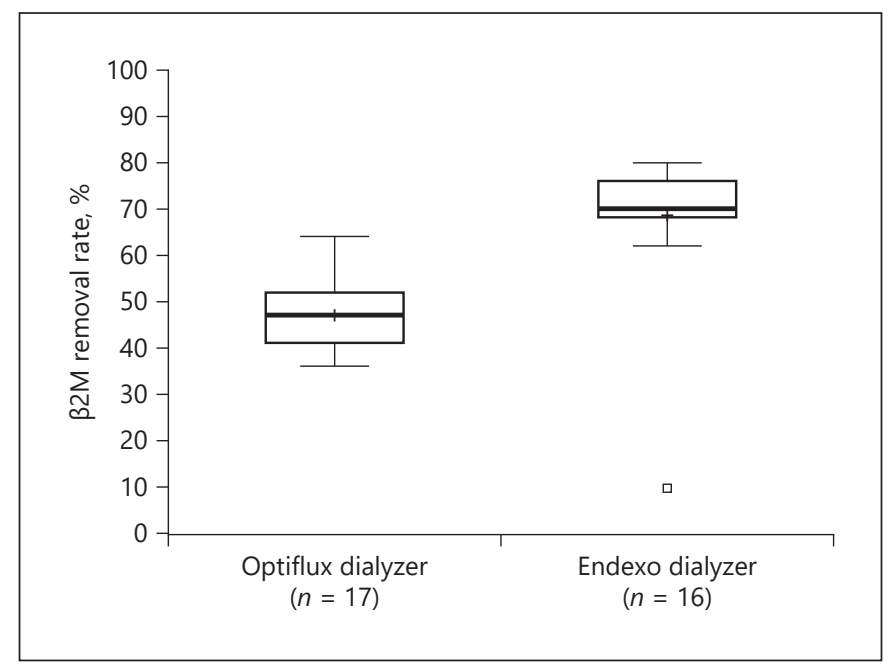

Fig. 2. Box plot of $\beta 2 \mathrm{M}$ removal rates by the dialyzer in the analysis population. Box represents interquartile range and median (horizontal line); whiskers represent adjacent values (i.e., highest value within Q3 + [1.5 $\times$ IQR $]$ and lowest value within Q1 + [1.5 $\times$ IQR]); +, mean value; $\square$, outlier value. Removal rate calculated as (pre-HD $\beta 2 \mathrm{M}$ - corrected post-HD $\beta 2 \mathrm{M}$ )/pre-HD $\beta 2 \mathrm{M} \times 100$. $\mathrm{HD}$, hemodialysis; IQR, interquartile range; $\beta 2 \mathrm{M}$, beta-2-microglobulin.

Endexo), and 3.91 (0.31) g/dL (visit 46; Endexo). Treatment with both dialyzers resulted in similar increases in serum albumin levels (Fig. 3).

During the 4 -week Optiflux period, 4 out of 23 patients reported a total of $7 \mathrm{AEs}$. This equates to a rate of $2.6 \mathrm{AEs}$ per 100 hemodialysis sessions. No single AE was reported by $>1$ patient, and none of the events were considered device related. During the Endexo period, 11 patients reported a total of $32 \mathrm{AEs}$, for a rate of 4.8 AEs per 100 hemodialysis sessions. As detailed in Table 3, the most commonly reported AEs (those occurring in $\geq 2$ patients) were headache, reported by 3 patients (16.7\%; 0.5 events per 100 hemodialysis sessions), and lethargy, hypotension, vomiting, and extremity pain, each reported by 2 patients (11.1\%; 0.3 events per 100 hemodialysis sessions). None of the AEs experienced in the Endexo period were considered device related. Overall, 3 serious AEs were reported (gastrointestinal hemorrhage, localized infection, and hypertensive emergency); all occurred during the Endexo period, and none were considered device or procedure related. No AEs led to study discontinuation, and there were no deaths during the study.

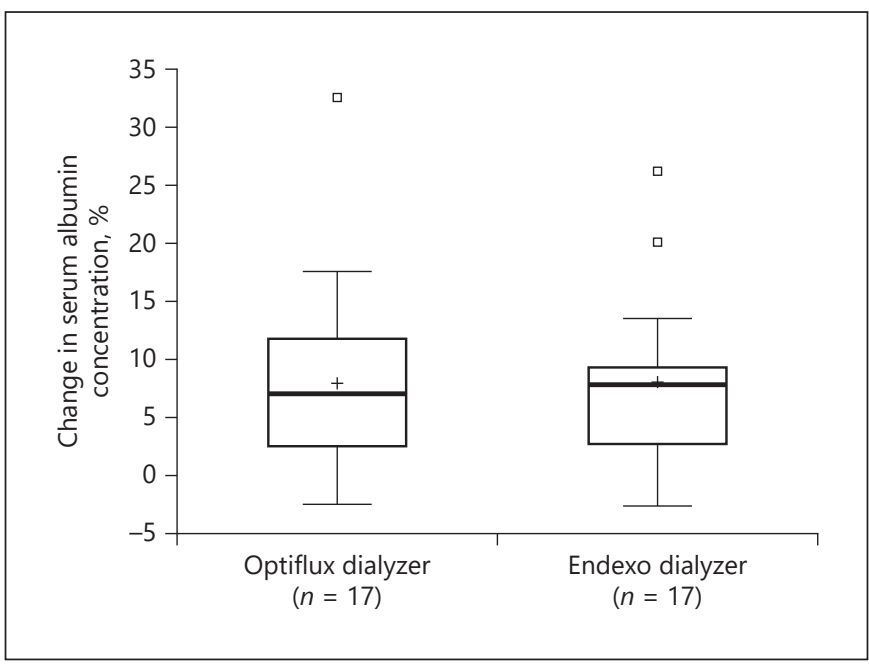

Fig. 3. Box plot of percentage changes in serum albumin concentration by the dialyzer (pre-HD to post-HD) in the analysis population. Box represents interquartile range and median (horizontal line); whiskers represent adjacent values (i.e., highest value within $\mathrm{Q} 3+[1.5 \times \mathrm{IQR}]$ and lowest value within Q1 + [1.5 $\times \mathrm{IQR}])$; +, mean value; $\square$, outlier value. Percent change $=$ (post-HD - pre$\mathrm{HD}) /$ pre-HD $\times 100 . \mathrm{HD}$, hemodialysis; IQR, interquartile range.

\section{Additional Endpoints}

The impact of dialysis on hematologic parameters (i.e., hemoglobin, hematocrit, and red blood cell count) was evaluated before and after the first study use of the dialyzer with Endexo. There was an absence of clinically significant changes in these parameters. Patients exhibited a mean (SD) hemoglobin increase of $0.49(0.93) \mathrm{g} / \mathrm{dL}$ after the first use of the dialyzer with Endexo. A mean (SD) platelet count decrease of $2.7 \%$ (5.70), from a predialysis mean (SD) of $198.94(55.85) \times 10^{3} / \mu \mathrm{L}$, was observed after the first use of the dialyzer with Endexo. Mean (SD) neutrophil counts were reduced from $4.28(1.79) \times 10^{3} / \mu \mathrm{L}$ (pre-HD) to $3.93(1.46) \times 10^{3} / \mu \mathrm{L}$ (post-HD) after the first session with the investigational dialyzer. Finally, a mean (SD) increase in red blood cells of $0.14 \times 10^{6} / \mu \mathrm{L}$ was observed after the first visit in the Endexo period.

No AEs related to laboratory test results were reported during the study. As expected, decreases in serum creatinine, phosphorus, and potassium levels were observed after dialysis. Mean liver enzyme (i.e., serum alanine aminotransferase [ALT] and serum aspartate aminotransferase [AST]) concentrations were numerically comparable at visits 13,22 , and 34 . At visit 46, 1 subject had elevated pre-HD serum ALT (271 U/L) and AST (273 U/L) values. 
Table 3. AEs reported during the Endexo period $(N=18)$

\begin{tabular}{lll}
\hline & Subjects, $n$ (\%) & Events, $n$ \\
\hline Headache & $3(16.7)$ & 3 \\
Asthenia & $2(11.1)$ & 2 \\
Hypotension & $2(11.1)$ & 2 \\
Pain in extremity & $2(11.1)$ & 2 \\
Vomiting & $2(11.1)$ & 2 \\
Arthralgia & $1(5.6)$ & 1 \\
Bowel movement irregularity & $1(5.6)$ & 1 \\
Cough & $1(5.6)$ & 1 \\
Diarrhea & $1(5.6)$ & 1 \\
Dyspepsia & $1(5.6)$ & 1 \\
Dyspnea & $1(5.6)$ & 1 \\
Gastrointestinal hemorrhage & $1(5.6)$ & 1 \\
Hypertension & $1(5.6)$ & 1 \\
Hypertensive emergency & $1(5.6)$ & 1 \\
Hypoesthesia & $1(5.6)$ & 1 \\
Influenza & $1(5.6)$ & 1 \\
Localized infection & $1(5.6)$ & 1 \\
Melena & $1(5.6)$ & 1 \\
Musculoskeletal chest pain & $1(5.6)$ & 1 \\
Musculoskeletal pain & $1(5.6)$ & 1 \\
Nasopharyngitis & $1(5.6)$ & 1 \\
Orbital edema & $1(5.6)$ & 1 \\
Tissue infiltration & $1(5.6)$ & 1 \\
Upper respiratory tract infection & $1(5.6)$ & 1 \\
Vascular access thrombosis & $1(5.6)$ & 1 \\
Wheezing & $1(5.6)$ & 1 \\
\hline
\end{tabular}

There were a total of 664 hemodialysis treatments from 18 subjects in the Endexo period. AEs, adverse events.

Because there were no protocol-driven modifications for hemodialysis treatment-related medications, investigators were permitted to alter medications based on clinical judgment and institutional guidelines. Relative to the Optiflux period, the mean dose of heparin was reduced by $11.2 \%$ during the Endexo period (mean [SD]: 4,507 $[3,349]$ IU vs. $3,760[2,471] \mathrm{IU})$. In a post hoc analysis that excluded 18 hemodialysis sessions from 6 patients with extremely high (i.e., $\geq 10,000 \mathrm{IU}$ ) and extremely low (i.e., $0 \mathrm{IU})$ recorded heparin doses, the dialyzer with Endexo was associated with a $3.7 \%$ mean reduction in heparin doses. All study sites administered unfractionated heparin as a single loading dose at the start of the dialysis treatment. Relative to treatment during the Optiflux period, patients received lower doses of ESA (12.0\% mean reduction) and higher doses of intravenous iron sucrose (13.4\% mean increase) during the Endexo period. In contrast, the mean administered doses of calcitriol, doxercalciferol, and saline were comparable across treatment periods (i.e., varied $<10 \%$ ).
There was no evidence of overt complement activation, as C5a and C3a levels remained largely unchanged after treatment with each dialyzer. A slight increase in sC5b-9 was observed for both dialyzers, with mean increases from 260 to $323 \mathrm{ng} / \mathrm{mL}$ during the Optiflux period and from 224 to $304 \mathrm{ng} / \mathrm{mL}$ during the Endexo period (Table 4).

Thrombus scoring was performed at the end of dialyzer use for every treatment session using a scale from grade 1 (good clear dialyzer, no detectable clotting) to 4 (total clotting of the dialyzer necessitating a stop in treatment or replacement of the dialyzer). The mean (SD) thrombus scores in the Optiflux and Endexo periods were $1.14(0.4)$ and 1.29 (0.52), respectively. A single grade 4 thrombus score was recorded during the study; it occurred during the Optiflux period.

\section{Discussion}

In this prospective, sequential, multicenter, open-label clinical study, the performance of the novel dialyzer with Endexo was comparable to the Optiflux dialyzer, and use of the dialyzer was well tolerated. The mean in vivo Kuf for the dialyzer was $15.85 \mathrm{~mL} / \mathrm{h} / \mathrm{mm} \mathrm{Hg}$. Although not evaluated in the present study, the in vitro Kuf of the dialyzer with Endexo is $81 \mathrm{~mL} / \mathrm{h} / \mathrm{mm} \mathrm{Hg}$ [11]. Discrepancies between the in vitro and in vivo performance of a dialyzer highlight pronounced differences in the testing modalities. Laboratory testing is performed under controlled conditions, often using animal blood with a constant hematocrit and specified total protein level, at UFRs between 600 and $1,800 \mathrm{~mL} / \mathrm{h}[7,12]$. In contrast, in vivo testing is subject to the interpatient variability of the cohort. For instance, at visit 13 in the present study, UFRs ranged from 310 to $1,020 \mathrm{~mL} / \mathrm{h}$ and TMP ranged from 20 to $80 \mathrm{~mm} \mathrm{Hg}$. It is also worth noting that Kuf itself generally varies during an HD session [12].

In the present study, the dialyzer with Endexo appeared to be $47 \%$ more efficient at removing $\beta 2 \mathrm{M}$ than the Optiflux dialyzer. The clinical relevance of this finding was not further examined in this short-term study. Accumulation of $\beta 2 \mathrm{M}$ in patients on long-term hemodialysis can result in deposition of amyloid (i.e., dialysisrelated amyloidosis) in musculoskeletal and cardiac tissue $[13,14]$. Higher clearance rates of $\beta 2 \mathrm{M}$ have been linked with reduced (or delayed) rates of dialysis-related amyloidosis [15]. Independent of duration of ESKD, diabetes, malnutrition, and chronic inflammation, higher $\beta 2 \mathrm{M}$ levels are predictive of increased mortality [16-18]. 
Table 4. Complement levels before and during the first session with each dialyzer

\begin{tabular}{|c|c|c|c|c|c|c|}
\hline & Optiflux & Endexo & Optiflux & Endexo & Optiflux & Endexo \\
\hline$N$ & 23 & 18 & 23 & 18 & 23 & 18 \\
\hline Mean (SD) & $1,606(1,621)$ & $1,319(887)$ & 8.55 (5.69) & $8.81(6.08)$ & $260(66)$ & $224(51)$ \\
\hline Median & 1,222 & 1,039 & 7.8 & 7.55 & 258 & 238 \\
\hline$N$ & 22 & 18 & 22 & 18 & 23 & 18 \\
\hline Mean (SD) & 1,355 (319) & $1,301(336)$ & $8.1(4.9)$ & $7.62(4.66)$ & $323(88)$ & $304(72)$ \\
\hline Median & 1,254 & 1,247 & 8.15 & 7.25 & 313 & 306 \\
\hline Range & $823-1,896$ & $780-2,172$ & $2.1-19.9$ & $1.6-15.7$ & $192-477$ & $206-476$ \\
\hline
\end{tabular}

Moreover, evidence suggests that greater removal of $\beta 2 \mathrm{M}$ may serve as a biomarker of reduced cardiovascular mortality among individuals receiving maintenance hemodialysis [16]. Although direct comparisons cannot be made, the $\beta 2 \mathrm{M}$ removal rate of the dialyzer with Endexo is greater than that traditionally reported for high-flux dialysis and comparable to that reported for medium-cutoff dialyzers [19]. The greater clearance of $\beta 2 \mathrm{M}$ observed with this novel dialyzer likely results from the modified surface characterization of the membrane. Addition of the fluorinated SMM provides a passive surface with low surface energy that can reduce adhesion and activation of blood proteins and platelets. In turn, this may make the membrane less susceptible to "fouling" and allow for greater middle molecule clearance. It is also plausible that differential adsorption of $\mathrm{B} 2 \mathrm{M}$ to the membrane may contribute to the observed differences in B2M removal [20]. Future assessment of dialysate-side B2M clearance could help clarify the mechanism(s) contributing to the higher B2M clearance rates associated with the dialyzer with Endexo. Future, longer-term studies could offer insight into what, if any, clinical implications might result from increased B2M clearance.

Both the Optiflux and the dialyzer with Endexo resulted in similar increases in serum albumin concentrations after hemodialysis. These increases were consistent with hemoconcentration as evidenced by mean hemoglobin increases of approximately $5 \%$ and a mean ultrafiltration volume removed of approximately $2.2 \mathrm{~L}$ with each dialyzer. Notably, predialysis albumin concentrations remained relatively constant across the 13-week Endexo period. This stability can be contrasted with recent data from a study of a medium-cutoff dialyzer demonstrating significant reductions (relative to a high-flux dialyzer) in serum albumin levels over a 3 -month period [21]. Among patients undergoing hemodialysis, reduced levels of serum albumin are a strong predictor of mortality, but the clinical significance of dialysis-associated reductions in albumin concentrations, in the absence of reductions in nutritional status, remains unclear $[21,22]$. The present trial was not of sufficient duration to assess long-term effects of dialyzer use on serum albumin levels or clinical outcomes. Additionally, information regarding protein intake was not captured during the trial, and dietary interventions were not addressed in the study protocol.

To reduce the risk of thrombosis and maintain circuit patency, patients on hemodialysis generally receive systemic anticoagulation with heparin. Investigators were not asked to follow any study-specific anticoagulant protocols. The reduction in heparin doses observed during the Endexo period was attenuated when a small number of sessions with outlier (recorded) heparin doses were excluded from the analysis. The clinical relevance of differences in thrombus scores is difficult to discern given the lack of protocoldriven systemic anticoagulation. The data from the present study suggest that the dialyzer with Endexo had no clinically relevant impact on hematologic parameters. The observed reductions in ESA doses during the Endexo period likely contributed to the observed increases in intravenous iron use and may reflect temporal changes in anemia management at the centers. Finally, there is a recognized association between reduced ESA needs and lower levels of inflammation as assessed by serum C-reactive protein levels [23]. Future studies should continue to explore the effects of the dialyzer with Endexo on anemia therapies and should examine inflammatory biomarkers. 


\section{Conclusions}

This study represents the first clinical study of a novel dialyzer containing the SMM Endexo blended into the membrane during manufacturing and was designed to align with FDA guidance on performance testing of new dialyzers [7]. More than 660 hemodialysis treatments were delivered during the Endexo period. The performance of the dialyzer with Endexo was generally comparable to that of the Optiflux dialyzer, and the dialyzer was well tolerated. The dialyzer with Endexo exhibited a high $\beta 2 \mathrm{M}$ removal rate (i.e., $68 \%$ with correction) and demonstrated no evidence of overt complement activation. Future studies will explore whether this dialyzer can be incorporated into a heparin-sparing hemodialysis system.

\section{Acknowledgement}

Medical writing and editing support was provided by Adam Perahia, MD, of NorthStar Strategic Consulting, LLC, via funding by the Fresenius Medical Care Renal Therapies Group.

\section{Statement of Ethics}

The clinical study protocol, written informed consent form, and all amendments that required approval were reviewed by an independent Institutional Review Board (Advarra Inc., Columbia, MD, USA).

\section{Conflict of Interest Statement}

A.A.Z., M.T., C.-H.H., S.A., C.M., and R.J.K. are or were employees of the Fresenius Medical Care Renal Therapies group during the study.

\section{Funding Sources}

The study was sponsored by the Fresenius Medical Care Renal Therapies Group, Waltham, MA, USA.

\section{Author Contributions}

J.M.M., D.S., and L.A.W. were principal investigators and contributed to the study conceptualization, patient enrollment and follow-up, and data analysis. A.A.Z. contributed to the study conceptualization, investigation, and methodology. M.T. contributed to the study conceptualization, investigation, methodology, resources, supervision, data analysis, and writing. C.H.H. contributed to the study conceptualization, investigation, methodology, data management and biostatistics supervision, and writing. S.A. contributed to the study conceptualization, methodology, and safety analysis. C.M. contributed to the study conceptualization, investigation, methodology, resources, supervision, data analysis, writing, and editing. R.J.K. contributed to the study conceptualization, investigation, resources, and supervision. All authors read and approved the final manuscript.

\section{References}

1 Liyanage T, Ninomiya T, Jha V, Neal B, Patrice HM, Okpechi I, et al. Worldwide access to treatment for end-stage kidney disease: a systematic review. Lancet. 2015 May; 385(9981):1975-82.

2 Haroon S, Davenport A. Choosing a dialyzer: what clinicians need to know. Hemodial Int. 2018 Oct;22(S2):S65-74.

3 Lopez-Donaire ML, Santerre JP. Surface modifying oligomers used to functionalize polymeric surfaces: consideration of blood contact applications. J Appl Polym Sci. 2014 Jan 7;131(14):40328.

4 AngioDynamics. BioFlo PICC with Endexo and PASV valve technology. Available from: https: //www.angiodynamics.com/products/23/BioFlo-PICC/.

5 AngioDynamics. BioFlo Duramax dialysis catheter with endexo technology. Available from: https: //www.angiodynamics.com/ products/27/BioFlo-DuraMax-DialysisCatheter-with-Endexo-Technology/.
6 Ho C-H, Fisher C, Mullon C. New polymeric dialysis membrane with Endexo ${ }^{\mathrm{ma}}$ surface modifying macromolecule. AJKD. 2020 Apr; 75(4):586.

7 US Department of Health and Human Services, Food and Drug Administration, Center for Devices and Radiological Health. Guidance for industry and CDRH reviewers: guidance for the content of premarket notifications for conventional and high permeability hemodialyzers. 1998 Aug 7. Available from: https: //www.fda.gov/media/72577/download. Accessed 2020 Mar 18.

8 Dorsch O, Krieter DH, Lemke HD, Fischer S, Melzer N, Sieder C, et al. A multi-center, prospective, open-label, 8-week study of certoparin for anticoagulation during maintenance hemodialysis-the membrane study. BMC Nephrol. 2012 Jun 28;13:50.

9 Laville M, Dorval M, Fort Ros J, Fay R, Cridlig J, Nortier JL, et al. Results of the HepZero study comparing heparin-grafted membrane and standard care show that heparin-grafted dialyzer is safe and easy to use for heparin-free dialysis. Kidney Int. 2014 Dec;86(6):1260-7.
10 Bergström J, Wehle B. No change in corrected beta 2-microglobulin concentration after cuprophane haemodialysis. Lancet. 1987 Mar; 1(8533):628-9.

11 Data on file. Fresenius Medical Care Renal Therapies Group LLC. Waltham, MA; 2019.

12 Ficheux A, Kerr PG, Brunet P, Argilés À. The ultrafiltration coefficient of a dialyser (KUF) is not a fixed value, and it follows a parabolic function: the new concept of KUF max. Nephrol Dial Transpl. 2011 Feb;26(2):63640.

13 Morris AD, Smith RN, Stone JR. The pathology and changing epidemiology of dialysisrelated cardiac beta-2 microglobulin amyloidosis. Cardiovasc Pathol. 2019 Sep-Oct;42: 30-5.

14 Fukuda K, Yamamoto H. Dialysis-related amyloidosis. Semin Musculoskelet Radiol. 2001 Jun;5(2):113-9.

15 Kaneko S, Yamagata K. Hemodialysis-related amyloidosis: is it still relevant? Semin Dial. 2018 Nov;31(6):612-8. 
16 Argyropoulos CP, Chen SS, Ng YH, Roumelioti ME, Shaffi K, Singh PP, et al. Rediscovering beta- 2 microglobulin as a biomarker across the spectrum of kidney diseases. Front Med. 2017 Jun;4:73.

17 Cheung AK, Rocco MV, Yan G, Leypoldt JK, Levin NW, Greene T, et al. Serum $\beta$ - 2 microglobulin levels predict mortality in dialysis patients: results of the HEMO Study. J Am Soc Nephrol. 2006 Feb;17(2):546-55.

18 Okuno S, Ishimura E, Kohno K, Fujino-Katoh Y, Maeno Y, Yamakawa T, et al. Serum beta2microglobulin level is a significant predictor of mortality in maintenance haemodialysis patients. Nephrol Dial Transpl. 2009 Feb; 24(2):571-7.
19 Masakane I, Sakurai K. Current approaches to middle molecule removal: room for innovation. Nephrol Dial Transpl. 2018 Oct;33(Suppl 1_3):iii12-21.

20 Roumelioti ME, Trietley G, Nolin TD, Ng YH, $\mathrm{Xu} \mathrm{Z}$, Alaini A, et al. Beta-2 microglobulin clearance in high-flux dialysis and convective dialysis modalities: a meta-analysis of published studies. Nephrol Dial Transpl. 2018 Jun;33(6):1025-39.
21 Belmouaz M, Bauwens M, Hauet T, Bossard V, Jamet P, Joly F, et al. Comparison of the removal of uraemic toxins with medium cutoff and high-flux dialysers: a randomized clinical trial. Nephrol Dial Transpl. 2020 Feb; 35(2):328-35.

$22 \mathrm{Ma} \mathrm{L}$, Zhao S. Risk factors for mortality in patients undergoing hemodialysis: a systematic review and meta-analysis. Int J Cardiol. 2017 Jul;238:151-8.

23 Chawla LS, Krishnan M. Causes and consequences of inflammation on anemia management in hemodialysis patients. Hemodial Int. 2009 Apr;13(2):222-34. 